\title{
Liquid-state paramagnetic relaxation from first principles
}

\author{
Jyrki Rantaharju* and Juha Vaara \\ NMR Research Unit, P.O. Box 3000, FIN-90014 University of Oulu, Finland
}

(Received 6 July 2016; published 19 October 2016)

\begin{abstract}
We simulate nuclear and electron spin relaxation rates in a paramagnetic system from first principles. Sampling a molecular dynamics trajectory with quantum-chemical calculations produces a time series of the instantaneous parameters of the relevant spin Hamiltonian. The Hamiltonians are, in turn, used to numerically solve the Liouville-von Neumann equation for the time evolution of the spin density matrix. We demonstrate the approach by studying the aqueous solution of the $\mathrm{Ni}^{2+}$ ion. Taking advantage of Kubo's theory, the spin-lattice $\left(T_{1}\right)$ and spin-spin $\left(T_{2}\right)$ relaxation rates are extracted from the simulations of the time dependence of the longitudinal and transverse magnetization, respectively. Good agreement with the available experimental data is obtained by the method.
\end{abstract}

DOI: 10.1103/PhysRevA.94.043413

\section{INTRODUCTION}

Quantitative understanding of the spin relaxation properties [1] of paramagnetic systems is fundamentally important for paramagnetic nuclear magnetic resonance (pNMR) and electron-spin- and electron-paramagnetic-resonance (ESR and EPR) spectroscopies. Both spectroscopic methods are very important in biomolecular and materials research [2-5]. Paramagnetic relaxation enhancement (PRE) in NMR has significant applications, for example, in the development of contrast agents for magnetic resonance imaging [6] and in studies of molecular structure [7]. Redfield relaxation theory [5,8-10], often used in magnetic resonance, is rarely applicable for paramagnetic systems. The stochastic Liouville equation $[11,12]$ (SLE) is the state-of-the-art method to simulate spin relaxation in paramagnetic systems. The most frequently used approach to solve SLE assumes that microscopic dynamics can be approximated in operator form, such as using diffusion operators and in the form of the Fokker-Planck SLE [11,12]. For paramagnetic systems, the operator form was developed in Ref. [13]. Application of SLE and its special cases, such as Swedish slow-motion theory [14,15], typically require empirical parameters either for the magnetic interaction Hamiltonian or for models of molecular motion. Parameterfree computations of the paramagnetic systems have so far been largely absent. Modern computational resources allow such first-principles approaches to be used in many areas of science to justify empirical models and fix their parameters.

In this paper, we take a nonempirical approach to solve the SLE in the Langevin form [16-19] and present first-principles computations in which the time evolution of the spin density matrix is governed by quantum-chemically calculated spin Hamiltonian. A time series of such Hamiltonians is sampled, in turn, from a molecular dynamics (MD) simulation trajectory. We simulate the EPR and pNMR relaxation rates of the aqueous solution of $\mathrm{Ni}^{2+}$ at room temperature. Earlier, the empirical parameters of the SLE were fitted to experimental results [20,21] for the example system, in Ref. [22]. Presently, we extend from our previous parameter-free study [23], where we simulated the EPR relaxation in this system, to PRE of the

\footnotetext{
*jyrki.rantaharju@oulu.fi
}

${ }^{1} \mathrm{H}$ and ${ }^{17} \mathrm{O}$ nuclei of the water molecules. To overcome the problem, in a simulation of finite length, posed by the much longer time scale of the nuclear spin relaxation as compared to that of the unpaired electrons, we employ the fact that the relaxation processes reach an asymptotic limit. We outline the argument behind Kubo's method [24,25] and apply it in the case of the $\mathrm{Ni}^{2+}(a q)$ system in this paper. The dynamical behavior of the ${ }^{1} \mathrm{H}$ and ${ }^{17} \mathrm{O}$ magnetization becomes asymptotic in the time scale of the simulation, whereas the magnetization of the effective unpaired electron spin $(S=1)$ of the $\mathrm{Ni}^{2+}$ ion decays all the way to the equilibrium within a time scale close to the correlation time of the relevant fluctuations. The length of the presently simulated spin dynamics (SD) trajectories is hundreds of ps, the correlation functions of the molecular fluctuations decay in the ps time scale, whereas the protons and ${ }^{17} \mathrm{O}$ nuclei relax in the ms and $\mu$ s time scales, respectively.

Characteristic to the present method is that the time series of the Hamiltonians is sampled with quantum-chemical (QC) calculations, which constitute by far the most expensive part of our simulation. For the present investigation, we chose the simple aqueous solution of $\mathrm{Ni}^{2+}$ ion as a well-studied prototypic system, which we have used also previously [23,26], and for which the time series of Hamiltonians were, therefore, available. More efficient quantum-chemical sampling techniques are being developed [27], based on preparametrized magnetic property hypersurfaces. Such sampling techniques will in the future allow the current first-principles spin relaxation method to be used to simulate also more complicated systems, with manageable computational cost.

\section{THEORY}

\section{A. Hamiltonian}

We consider our example system, the aqueous solution of the $\mathrm{Ni}^{2+}$ ion, as an ensemble of electron and nuclear spins with the appropriate spin Hamiltonian defined as

$$
\begin{aligned}
\hat{H}(t)= & \mu_{\mathrm{B}} \hat{\boldsymbol{S}} \cdot \boldsymbol{g}(t) \cdot \boldsymbol{B}+\gamma \hbar \hat{\boldsymbol{I}} \cdot[\mathbf{1}-\boldsymbol{\sigma}(t)] \cdot \boldsymbol{B} \\
& +\hat{\boldsymbol{S}} \cdot \boldsymbol{D}(t) \cdot \hat{\boldsymbol{S}}+\hat{\boldsymbol{S}} \cdot \boldsymbol{A}(t) \cdot \hat{\boldsymbol{I}},
\end{aligned}
$$

where $\hat{\boldsymbol{S}}$ stands for electron spin vector and $\hat{\boldsymbol{I}}$ for the nuclear spin vector of an individual water proton or ${ }^{17} \mathrm{O}$ nucleus. In the terms of the Hamiltonian we include the $g$ tensor 
(g) parametrizing the electronic Zeeman interaction with the external magnetic field $\boldsymbol{B}$, the nuclear shielding tensor $\boldsymbol{\sigma}$ modifying the corresponding nuclear Zeeman interaction, the zero-field splitting (ZFS) tensor $\boldsymbol{D}$, and the hyperfine coupling (HFC) tensor $\boldsymbol{A}$, arising from the spin-orbit coupling and magnetic interaction between the unpaired electron and the nuclear spin, respectively. In the Hamiltonian (1), $\mu_{\mathrm{B}}$ is the Bohr magneton and $\gamma$ is the nuclear gyromagnetic ratio. We assume that both direct and indirect spin-spin couplings between the nuclei are insignificant for PRE, and we also ignore the quadrupole coupling of the spin- $\frac{5}{2}{ }^{17} \mathrm{O}$ nucleus, whose fluctuations dominate ${ }^{17} \mathrm{O}$ relaxation in diamagnetic systems (see, e.g., [28] and references therein). The ensemble and, assuming ergodicity, time average of the Hamiltonian $\langle\hat{H}(t)\rangle$ equals the sum of the Zeeman terms $\hat{H}_{z}$ spanned by the components of the electron and nuclear spin vectors along $\boldsymbol{B} . \hat{H}_{z}$ is time independent and we define the interaction Hamiltonian as $\hat{H}_{I}(t)=\hat{H}(t)-\hat{H}_{z}$.

\section{B. Spin dynamics}

The density operator for an individual member of the ensemble, i.e., a pure state, is defined as $\hat{\rho}=|\Psi\rangle\langle\Psi|$, where $|\Psi\rangle$ is the state of the spin system. The dynamical behavior of $\hat{\rho}$ is determined by the Liouville-von Neumann equation $[29,30]$

$$
\frac{d \hat{\rho}(t)}{d t}=\hat{\hat{L}}(t) \hat{\rho}(t)
$$

where the Liouvillian $\hat{\hat{L}}(t)$ is a superoperator defined as (operating on the operator $\hat{c}$ )

$$
\hat{\hat{L}}(t) \hat{c}=-\frac{i}{\hbar}[\hat{H}(t), \hat{c}] .
$$

Using $\delta_{i}$ to denote the $i$ th discrete time step, and defining $t_{j}=\sum_{i=1}^{j} \delta_{i} \forall j$, we can approximate

$$
\hat{\rho}(t) \approx e^{\hat{\hat{L}\left(t_{n}\right) \delta_{n}}} \ldots e^{\hat{\hat{L}}\left(t_{1}\right) \delta_{1}} \hat{\rho}(0) \equiv \hat{\rho}_{n}(t) .
$$

Letting $\delta \rightarrow 0$, and, thus, the number of time steps $n \rightarrow \infty$, $\hat{\rho}_{n}(t)$ becomes the exact solution of Eq. (2).

Assuming that all the spins in the ensemble are initially in the same state $\hat{\rho}(0)$, the ensemble average of the density operator at time $t$ can be expressed as

$$
\langle\hat{\rho}(t)\rangle=\lim _{\max \left(\delta_{i}\right) \rightarrow 0}\left\langle e^{\hat{\hat{L}}\left(t_{n}\right) \delta_{n}} \ldots e^{\hat{\hat{L}}\left(t_{1}\right) \delta_{1}}\right\rangle \hat{\rho}(0) .
$$

We define

$$
\hat{\mathscr{L}}(t)=\lim _{\max \left(\delta_{i}\right) \rightarrow 0} \ln \left[\left\langlee^{\hat{\hat{L}}\left(t_{n}\right) \delta_{n}} \ldots e^{\left.\left.\hat{\hat{L}\left(t_{1}\right) \delta_{1}}\right\rangle\right]}\right.\right.
$$

to yield exponential propagator $e^{\hat{\mathscr{L}}(t)}$ for the ensembleaveraged density operator.

The operators and superoperators can be cast into the rotating frame, respectively, as

$$
\hat{O}^{r}(t)=e^{-\hat{\hat{L}}_{z} t} \hat{O}(t) ; \hat{\hat{O}}^{r}(t)=e^{-\hat{\hat{L}}_{z} t} \hat{\hat{O}}(t) e^{\hat{\hat{L}}_{z} t},
$$

where $\hat{\hat{L}}_{z}$ is the Liouvillian form [as in Eq. (3)] of $\hat{H}_{z}$. The Liouville-von Neumann equation (2) for the density operator in the rotating frame (interaction picture) reads as then

$$
\frac{d \hat{\rho}^{r}(t)}{d t}=\hat{\hat{L}}_{I}^{r}(t) \hat{\rho}^{r}(t)
$$

where $\hat{\hat{L}}_{I}^{r}(t)$, the Liouvillian form of $\hat{H}_{I}(t)$ in the rotating frame, is the Liouvillian in the interaction picture [31]. One can show that $\hat{\hat{L}}_{I}(t)=\hat{\hat{L}}(t)-\hat{\hat{L}}_{z}$ and $\hat{\hat{L}}_{I}^{r}(t)=\hat{\hat{L}}^{r}(t)-\hat{\hat{L}}_{z}$. Thus, the superpropagator for the ensemble-averaged density operator in the rotating frame equals $e^{\hat{\mathscr{L}}_{I}^{r}(t)}$, where

$$
\hat{\mathscr{L}}_{I}^{r}(t)=\lim _{\max \left(\delta_{i}\right) \rightarrow 0} \ln \left[\left\langle e^{\hat{\hat{L}}_{I}^{r}\left(t_{n}\right) \delta_{n}} \ldots e^{\hat{\hat{L}}_{I}^{r}\left(t_{1}\right) \delta_{1}}\right\rangle\right] .
$$

Hence, $\left\langle\hat{\rho}^{r}(t)\right\rangle=e^{\hat{\mathscr{L}}_{I}^{r}(t)} \hat{\rho}(0)$, and from the simple connection $\left\langle\hat{\rho}^{r}(t)\right\rangle=e^{-\hat{\hat{L}}_{z} t}\langle\hat{\rho}(t)\rangle$, we have the equality

$$
e^{\hat{\mathscr{L}}_{I}^{r}(t)}=e^{-\hat{\hat{L}}_{z} t} e^{\hat{\mathscr{L}}(t)} .
$$

The operators of the spin system can be represented in the bra-ket vector notation in the so-called shift and $z$-operator basis (SZOB), which consists of the normalized shift, $z$, and unit operators [32]

$$
\hat{S}_{z}, \hat{S}_{+}, \hat{S}_{-}, \hat{I}_{z}, \hat{I}_{+}, \hat{I}_{-} \text {, and } \hat{1},
$$

as well as certain normalized linear combinations of the products of these operators (the shift operators are defined as, e.g., $\left.\hat{S}_{+}=\hat{S}_{x}+i \hat{S}_{y}, \hat{S}_{-}=\hat{S}_{x}-i \hat{S}_{y}\right)$. The diagonal and offdiagonal elements of $e^{\hat{\mathscr{L}}(t)}$ are comprised of the autocorrelation and cross-correlation functions, respectively, of the operators of the basis.

With the definition $|\hat{O}(t)\rangle=e^{\hat{\hat{L}}(t)}|\hat{O}(0)\rangle$, the ensembleaveraged bra-ket operator products can be expressed as

$$
\langle\hat{O}(0) \mid \hat{O}(t)\rangle=\frac{\operatorname{Tr}\langle\hat{O}(0) \hat{O}(t)\rangle}{\operatorname{Tr}\langle\hat{O}(0) \hat{O}(0)\rangle},
$$

where $\langle\ldots\rangle$ denotes the ensemble average.

\section{Asymptotic limit}

In the definition of $\hat{\mathscr{L}}_{I}^{r}(t)$ [Eq. (9)], we may expand the natural logarithm as a Taylor series in terms of the variables $\delta_{i}$, around 0 . Then, it follows from Kubo's theorem [24] that taking the limit $\max \left(\delta_{i}\right) \rightarrow 0$ leads to the so-called generalized cumulant expansion (GCE). The GCE is the series

$$
\hat{\mathscr{L}}_{I}^{r}(t)=\sum_{i=1}^{\infty} \hat{\hat{K}}_{i}^{r}(t),
$$

where

$$
\begin{aligned}
\hat{\hat{K}}_{i}^{r}(t)= & \int_{0}^{t} d t_{i} \int_{0}^{t_{i}} d t_{i-1} \ldots \\
& \times \int_{0}^{t_{2}} d t_{1}\left\langle\hat{\hat{L}}_{I}^{r}\left(t_{i}\right) \hat{\hat{L}}_{I}^{r}\left(t_{i-1}\right) \ldots \hat{\hat{L}}_{I}^{r}\left(t_{1}\right)\right\rangle_{c} .
\end{aligned}
$$

In the definition, $\langle\ldots\rangle_{c}$ is the cumulant average [24,31] over the ensemble, defined via the generating function [33]

$$
\begin{aligned}
& \left\langle\hat{\hat{L}}_{I}^{r}\left(t_{k}\right) \ldots \hat{\hat{L}}_{I}^{r}\left(t_{1}\right)\right\rangle_{c} \\
& \quad=\left.\frac{\partial^{k}}{\partial \delta_{1} \ldots \partial \delta_{k}}\left[\ln \left\langle e^{\hat{\hat{L}}_{I}^{r}\left(t_{k}\right) \delta_{k}} \ldots e^{\hat{\hat{L}}_{I}^{r}\left(t_{1}\right) \delta_{1}}\right\rangle\right]\right|_{\delta_{i}=0 \forall i} .
\end{aligned}
$$


In the interaction picture, the cumulant average has the property

$$
\left\langle\hat{\hat{L}}_{I}^{r}\left(t_{1}\right)\right\rangle_{c}=\left\langle\hat{\hat{L}}_{I}^{r}\left(t_{1}\right)\right\rangle=0 .
$$

Thus, the first term of the GCE vanishes. We define $\tau$ as the smallest time for which $\left\langle\hat{\hat{L}}_{I}^{r}(0) \mid \hat{\hat{L}}_{I}^{r}(t)\right\rangle \approx 0$ for $t>\tau$, where the inner product of the superoperators is defined analogous to Eq. (12). This means that, for $t>\tau, \hat{\hat{L}}_{I}^{r}(t)$ does not correlate significantly with $\hat{\hat{L}}_{I}^{r}(0)$. Now,

$$
\int_{0}^{\tau}\left\langle\hat{\hat{L}}_{I}^{r}(0) \mid \hat{\hat{L}}_{I}^{r}(t)\right\rangle d t \approx \int_{0}^{\infty}\left\langle\hat{\hat{L}}_{I}^{r}(0) \mid \hat{\hat{L}}_{I}^{r}(t)\right\rangle d t=\tau_{c},
$$

where $\tau_{c}$ is the correlation time. Because of the chronological time ordering in the definition (14), $t_{i} \geqslant t_{j}$ if and only if $i>j$, we see that, in case $t_{i}-t_{i-1}>\tau$,

$$
\begin{aligned}
& \ln \left\langle e^{\hat{\hat{L}}_{I}^{r}\left(t_{n}\right) \delta_{n}} \ldots e^{\hat{\hat{L}}_{I}^{r}\left(t_{i}\right) \delta_{i}} e^{\hat{\hat{L}}_{I}^{r}\left(t_{i-1}\right) \delta_{i-1}} \ldots e^{\hat{\hat{L}}_{I}^{r}\left(t_{1}\right) \delta_{1}}\right\rangle \\
& \quad=\ln \left\langle e^{\hat{\hat{L}}_{I}^{r}\left(t_{n}\right) \delta_{n}} \ldots e^{\hat{\hat{L}}_{I}^{r}\left(t_{i}\right) \delta_{i}}\right\rangle+\ln \left\langle e^{\hat{\hat{L}}_{I}^{r}\left(t_{i-1}\right) \delta_{i-1}} \ldots e^{\hat{\hat{L}}_{I}^{r}\left(t_{1}\right) \delta_{1}}\right\rangle .
\end{aligned}
$$

Hence, the cumulant average vanishes in this case. As shown by Kubo [11,24], $\hat{\hat{K}}_{i}^{r}(t)$ starts to behave asymptotically at $t>\tau_{i}$, where $\tau_{i}=(i-1) \tau$. We assume that the GCE converges and, consequently, as shown in the Appendix A, provided that $\hat{K}_{j}^{r}(t)$ is the highest significant term in the GCE,

$$
\left.\hat{\hat{L}}_{I}^{r}(t)\right|_{t>\tau_{j}}=\hat{\hat{A}} t+\hat{\hat{\mathscr{B}}}
$$

with

$$
\begin{aligned}
& \hat{\hat{\mathscr{A}}}=e^{-\hat{\hat{L}}_{z}\left(t-\tau_{j}\right)} \dot{\hat{\mathscr{L}}}_{I}^{r}\left(\tau_{j}\right) e^{\hat{\hat{L}}_{z}\left(t-\tau_{j}\right)}, \\
& \hat{\hat{\mathscr{B}}}=\left[\hat{\mathscr{L}}_{I}^{r}\left(\tau_{j}\right)-e^{-\hat{\hat{L}}_{z}\left(t-\tau_{j}\right)} \dot{\hat{\mathscr{L}}}_{I}^{r}\left(\tau_{j}\right) e^{\hat{\hat{L}}_{z}\left(t-\tau_{j}\right)} \tau_{j}\right] \text {, }
\end{aligned}
$$

where the dot over $\hat{\mathscr{L}}_{I}^{r}$ is used to express the explicit time derivative $\frac{\partial}{\partial t}$. $\hat{\mathscr{A}}$ and $\hat{\hat{\mathscr{B}}}$ are independent of time in the explicit sense. However, the implicit time dependence due to the rotating frame is shown in Eq. (20) for convenience. In relaxation processes where $\tau_{j}$ is small as compared to the anticipated relaxation time, the effect of the $\hat{\hat{\mathscr{B}}}$ term is insignificant. In this case, the effective superpropagator for the ensemble-averaged density operator is $e^{-\hat{\hat{L}}_{z}\left(t-\tau_{j}\right)} e^{\dot{\hat{\mathscr{L}}}_{I}^{r}\left(\tau_{j}\right) t} e^{\hat{\hat{L}}_{z}\left(t-\tau_{j}\right)}$. The double-sided rotation has no effect on relaxation, which, consequently, is described simply by $e^{\dot{\hat{\mathcal{L}}}_{I}^{r}\left(\tau_{j}\right) t}$.

In the GCE, we may choose to include in $\hat{\hat{K}}_{i}^{r}(t)$ terms only up to the second one $\hat{\hat{K}}_{2}^{r}(t)$ to yield the second-order approximation for $e^{\hat{\mathscr{L}}_{I}^{r}(t)}$. Because of Eq. (16) and the stationarity of the ensemble, we see that

$$
\begin{aligned}
\hat{\hat{K}}_{2}^{r}(t) & =\int_{0}^{t} d t_{2} \int_{0}^{t_{2}} d t_{1}\left\langle\hat{\hat{L}}_{I}^{r}\left(t_{2}\right) \hat{\hat{L}}_{I}^{r}\left(t_{1}\right)\right\rangle \\
& =\int_{0}^{t} d t_{2} \int_{t_{2}}^{2 t_{2}} d t_{1}\left\langle\hat{\hat{L}}_{I}^{r}\left(t_{2}\right) \hat{\hat{L}}_{I}^{r}\left(t_{1}\right)\right\rangle,
\end{aligned}
$$

where the last expression of Eq. (21) is used in the practical simulation. In the regime where the Redfield theory is valid, the second-order term is the only significant term $(j=2)$ :

$$
\begin{aligned}
\dot{\hat{\hat{L}}}_{I}^{r}\left(\tau_{2}\right)=\dot{\hat{\hat{K}}}_{2}^{r}\left(\tau_{2}\right) & =\int_{0}^{\tau_{2}} d t_{1}\left\langle\hat{\hat{L}}_{I}^{r}\left(\tau_{2}\right) \hat{\hat{L}}_{I}^{r}\left(t_{1}\right)\right\rangle \\
& =\int_{0}^{\tau_{2}} d t_{1}\left\langle\hat{\hat{L}}_{I}^{r}(0) \hat{\hat{L}}_{I}^{r}\left(t_{1}\right)\right\rangle,
\end{aligned}
$$

and the $\hat{\hat{\mathscr{B}}}$ term is insignificant. The last expression of Eq. (22) is the typical form of the Redfield relaxation matrix.

\section{Spin dynamics simulations}

We extract a time series of instantaneous Hamiltonians $\left\{\hat{H}_{i}\right\}_{i=1}^{l}$, with the adjacent configurations separated by time step $\delta=48 \mathrm{fs}$, from a 750-ps-long production part of MD simulation trajectory of $\mathrm{Ni}^{2+}(a q)$, with the snapshots sampled using quantum-chemical electronic structure calculations [23] of the parameters of the spin Hamiltonian (1). The series consists of $l=15625$ time steps, and determines the time dependence of a single spin system in the ensemble. By assuming ergodicity and calculating the ensemble average as a time average over the series of Hamiltonians, we approximate

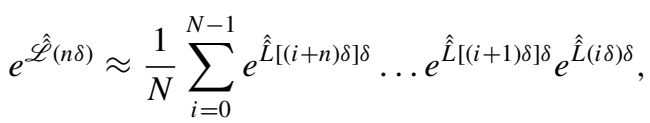

where $N$ is the normalization factor. At each point in time $n \delta$, we average over as many subseries $\hat{H}_{i}, \ldots, \hat{H}_{i+n}$ as can be extracted from the series $\left\{\hat{H}_{i}\right\}_{i=1}^{l}$. Thus, $N$ depends on $n$ as well as $l$. For each value of $n$ at which we calculate $e^{\hat{\mathscr{L}}(n \delta)}$, $(1 / N) \sum_{i=1}^{N} \hat{H}_{i}$ should ideally provide a good approximation of $\hat{H}_{z}$. In practice, the statistical quality of the simulated magnetization decreases for larger $n$ values. Ideally, $\delta$ should be small enough to capture all the significant fluctuations. By setting $\delta$ to be 48 fs we yield sampling frequency corresponding to the electron Zeeman frequency at $708.1 \mathrm{~T}$ external magnetic field. In a 1-T external field, the Zeeman frequency of the electron is $29.4207 \mathrm{GHz}$, that of the protons 42.5802 MHz and, for oxygen, 5.87229 MHz.

We obtain $\hat{\mathscr{L}}_{I}^{r}(n \delta)$ by applying rotation operator $e^{-\hat{\hat{L}}_{z} n \delta}$, from the left-hand side, to the numerical approximation (23) [see Eq. (10)], and finally taking the natural logarithm. We calculate the normalized spin-lattice (longitudinal, $z$ axis) and spin-spin (transverse, $x$ or $y$ axis) magnetization decay as

$$
\frac{\sigma_{\epsilon}^{r}(t)}{\sigma_{\epsilon}^{r}(0)}=\left\langle\hat{\sigma}_{\epsilon}(0) \mid \hat{\sigma}_{\epsilon}^{r}(t)\right\rangle=\left\langle\hat{\sigma}_{\epsilon}(0)\left|e^{\hat{\mathscr{L}}_{I}^{r}(n \delta)}\right| \hat{\sigma}_{\epsilon}(0)\right\rangle,
$$

where $\sigma_{\epsilon}$ stands for the Cartesian $\epsilon$ component $(\epsilon=x, y, z)$ of the electron, proton, or oxygen spin. With the available statistics, the off-diagonal elements of the calculated $\hat{\mathscr{L}}_{I}^{r}(n \delta)$ are unfortunately rather noisy in the SZOB operator basis. To work around this, we assume that $\hat{\mathscr{L}}_{I}^{r}(n \delta)$ is diagonal after the asymptotic limit is reached, i.e., the magnetization decay assumes a single-exponential form (the normalized $\sigma_{z}$ and $\sigma_{ \pm}$ operators belong to SZOB).

We also study the effect of the second-order [Eq. (21)] contribution to the diagonal of $\hat{\mathscr{L}}_{I}^{r}(t)$, by straightforwardly 
calculating

$$
\begin{aligned}
\hat{\hat{K}}_{2}^{r}(n \delta) \approx & \frac{\delta^{2}}{N} \sum_{i=0}^{N-1} \sum_{m_{2}=1}^{n} \sum_{m_{1}=m_{2}}^{2 m_{2}-1} \hat{\hat{L}}_{I}^{r}(i \delta) \hat{\hat{L}}_{I}^{r}\left[\left(m_{1}-m_{2}+i\right) \delta\right] \\
& -\frac{\delta}{2 N} \sum_{i=0}^{N-1} \hat{\hat{L}}_{I}^{r}(i \delta)^{2} n \delta
\end{aligned}
$$

where $N$ is the normalization factor explained above. For the approximation to remain valid, we must assume ergodicity of the ensemble in the rotating frame. The first term on the the right-hand side of Eq. (25) is a practical approximate form of the last expression in Eq. (21), whereas the second term of Eq. (25) is a correction that arises due to the finite length of the time step, as explained in Appendix B.

In the calculations of the instantaneous spin Hamiltonian parameters we take only into account the first coordination sphere of the ion, consisting of six water molecules. The $\mathrm{Ni}\left(\mathrm{H}_{2} \mathrm{O}\right)_{6}^{2+}$ entity has an isotropic rotational distribution in the liquid phase. Also, the dynamic behavior of the $g$ and ZFS tensors is independent of the direction of $\mathbf{B}$ with respect to the molecular system. Thus, similarly as in our previous work [23], we gain threefold improved statistics by rotating the molecular property tensors so that the magnetic field is, in turn, along each of the Cartesian $x, y$, and $z$ axes of the MD simulation box. The spin dynamics trajectories of the longitudinal and transverse components of magnetization $\mathbf{M}$, obtained with the different choices of the direction of $\mathbf{B}$, are averaged over. In the cases of the ${ }^{1} \mathrm{H}$ and ${ }^{17} \mathrm{O}$ simulations, we average over all the corresponding nuclei in the first solvation shell of the ion. The $g$, ZFS, and hyperfine coupling tensors were calculated in the same way and at the same level of theory as described in Ref. [26].

We used the SPINDYNAMICA [32] software package for Mathematica [34] as a platform for the spin dynamics simulations.

\section{RESULTS AND DISCUSSION}

Both the spin-lattice and spin-spin magnetization decays assume the single-exponential form

$$
\frac{\sigma_{z}^{r}(t)}{\sigma_{z}^{r}(0)}=e^{-a_{1} t+b_{1}} ; \quad \frac{\sigma_{x, y}^{r}(t)}{\sigma_{x, y}^{r}(0)}=e^{-a_{2} t+b_{2}}
$$

after reaching the asymptotic limit since $\hat{\mathscr{L}}_{I}^{r}(n \delta)$ is approximated to be diagonal. We extracted the spin-lattice $\left(a_{1}=\right.$ $\left.1 / T_{1}\right)$ and spin-spin $\left(a_{2}=1 / T_{2}\right)$ relaxation rates and the corresponding constants $b_{1}$ and $b_{2}$, by least-square fitting with the FINDFIT routine of Mathematica.

In analogy to the case of the full simulation, the secondorder contribution to relaxation rates $a_{1,2}$ (and constants $b_{1,2}$ ) assume the form

$$
\begin{aligned}
& -a_{1}^{(2)} t+b_{1}^{(2)}=\left\langle\hat{\sigma}_{z}(0)\left|\hat{\hat{K}}_{2}^{r}(t)\right| \hat{\sigma}_{z}(0)\right\rangle, \\
& -a_{2}^{(2)} t+b_{2}^{(2)}=\left\langle\hat{\sigma}_{x, y}(0)\left|\hat{\hat{K}}_{2}^{r}(t)\right| \hat{\sigma}_{x, y}(0)\right\rangle
\end{aligned}
$$

after the second-order part has reached the asymptotic limit. The spin-lattice $a_{1}^{(2)}$ and spin-spin $a_{2}^{(2)}$ relaxation rates

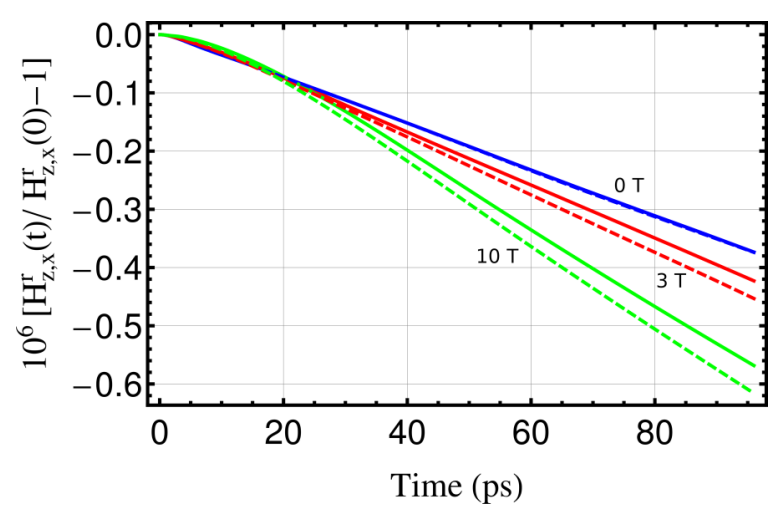

FIG. 1. Simulated proton magnetization decays $H_{z}^{r}(t) / H_{z}^{r}(0)$ (solid lines) and $H_{x}^{r}(t) / H_{x}^{r}(0)$ (dashed lines) defined by Eq. (24) at $0 \mathrm{~T}$ (blue), $3 \mathrm{~T}$ (red), and $10 \mathrm{~T}$ (green) external magnetic-field strengths, in $\mathrm{Ni}^{+2}(a q)$ at $300 \mathrm{~K}$.

and the constants $b_{1}^{(2)}$ and $b_{2}^{(2)}$ were again extracted by fitting.

\section{A. Relaxation of the water protons}

Figure 1 shows the longitudinal and transverse magnetization decay of the proton at 0,3 , and $10 \mathrm{~T}$ external magnetic-field strengths. The time scale of the simulation is more than sufficient for the proton magnetization decay to assume the linear form $1+b_{1,2}-a_{1,2} t$, which happens after circa 40 ps. The relaxation rate increases with $B$ and at these finite field values the longitudinal relaxation occurs slower than the transverse relaxation.

Figure 2 shows the extracted ${ }^{1} \mathrm{H}$ spin-lattice and spin-spin relaxation rates $a_{1}$ and $a_{2}$, respectively. It also includes a comparison with experimental results $[20,21]$ for the first-shell contribution to $a_{1}$ at temperatures of 324 and $344 \mathrm{~K}$. The simulated data show a flat region at small values of $B$, where the relaxation rates do not significantly depend on the magnetic-field strength. At higher field values, beyond $1 \mathrm{~T}$, $a_{1}$ turns to a rapid increase. Both qualitative observations

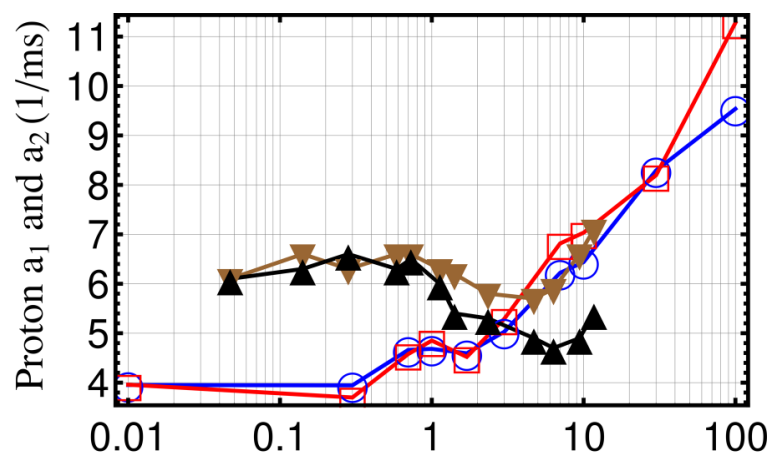

External Magnetic Field Strength B (T)

FIG. 2. Simulated $a_{1}$ (spin-lattice, blue open circles) and $a_{2}$ (spinspin, red open squares) relaxation rate of the first-shell proton spins in $\mathrm{Ni}^{2+}(a q)$ as a function of the external magnetic-field strength, at $300 \mathrm{~K}$. The triangles represent experimental $a_{1}$ results [20,21], black (arrow up) at $344 \mathrm{~K}$ and brown (arrow down) at $324 \mathrm{~K}$. 
are in agreement with experimental findings [20,21]. It is noteworthy that we reach a semiquantitative agreement with experiment from first principles. The order of magnitude of the pNMR relaxation rates is reproduced here without adjustable parameters either in the motional model or in the magnetic interactions.

In the detailed behavior of the $a_{1}$ relaxation rate at intermediate field strengths, our simulated data differ from the experiments. The measurements, albeit performed at slightly higher temperatures than the $300 \mathrm{~K}$ of the simulation, feature a region of minimum in the spin-lattice relaxation rate at $B$ around a couple of Teslas. Such a clear minimum at that field strength is not reproduced in our results based on the magnetization decay curves obtained from our limited statistical sample. Our data, however, feature a somewhat less distinct minimum above $1 \mathrm{~T}$. The statistics used in our SD simulation were extracted from a MD simulation of a single $\mathrm{Ni}^{2+}$ ion surrounded by water molecules. Such a limited sample size is dictated by the fact that the QC calculation of the spin Hamiltonian parameters of the instantaneous simulation snapshots is very time consuming. In the long term, such simulations may be performed using accurate preparametrized magnetic property hypersurfaces, which no longer require brute-force QC sampling of instantaneous configurations [27]. This will enable both using a smaller time step $\delta$ and a longer total SD simulation length, which improve reproduction of the physical fluctuations contributing to relaxation as well as statistical averaging.

We include in the Supplemental Material [35] figures of the $B$ dependence of the relaxation rates of the individual protons in the first solvation shell of the $\mathrm{Ni}^{2+}$ ion. These relaxation rates differ clearly from each other, with many of them showing minimum features akin to the experimental behavior, at the intermediate field strengths. This and the fact that the simulated $a_{2}$ does not always exceed $a_{1}$ in magnitude imply that the present statistical sample remains insufficient for drawing detailed quantitative conclusions about the magnetic-field dependence, despite the remarkable success in reproducing the order of magnitude of the relaxation rates, as well as the qualitative features described above.

ZFS (the $\boldsymbol{D}$ tensor) and the electronic Zeeman ( $g$ tensor) interactions give only off-diagonal contributions to the nuclear spin relaxation through the second-order term of the generalized cumulant expansion. From the diagonal assumption (i.e., that the relaxation is assumed single exponential) it, thus, follows that the second-order nuclear spin relaxation rates are only contributed to by the HFC (the $\boldsymbol{A}$ tensor) and nuclear Zeeman (the $\sigma$ tensor) interactions. Figure 3 shows the simulated second-order proton spin-lattice and spin-spin relaxation rates $a_{1}^{(2)}$ and $a_{2}^{(2)}$, respectively, as functions of $B$ at $300 \mathrm{~K}$. In contrast to both the full simulation and experiment, the second-order rates decrease monotonically as functions of $B$. At intermediate values of $B, a_{2}^{(2)}<a_{1}^{(2)}$, which is one indicator of the insufficiency of the second-order contribution. It is, however, noteworthy that the second-order rates approach those of the full simulation at the largest simulated field strengths, indicating that the second-order term in the GCE becomes increasingly dominant, as $B$ grows. Related to that, Fig. 3 shows the indispensable role of the higher- than second-order terms of the GCE for ${ }^{1} \mathrm{H}$ relaxation

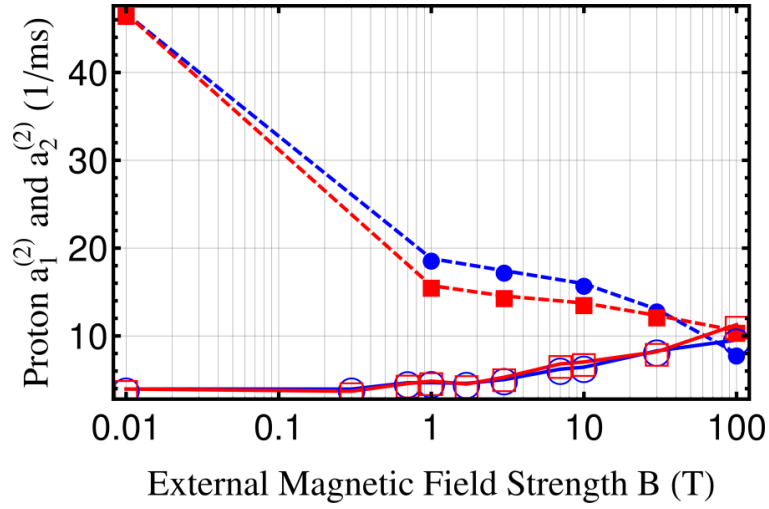

FIG. 3. Simulated second-order first-shell proton relaxation rates $a_{1}^{(2)}$ (blue closed circles, dashed line) and $a_{2}^{(2)}$ (red closed squares, dashed line) in $\mathrm{Ni}^{2+}(a q)$, compared to the results of the full simulation: $a_{1}$ (blue open circles, solid line) and $a_{2}$ (red open squares, solid line), at $300 \mathrm{~K}$.

at the experimentally relevant, smaller magnetic fields. The correction term (latter term) in Eq. (25) is insignificantly small in the second-order nuclear relaxation rates.

The second-order theory is insufficient for describing the relaxation of the proton spin because the HFC interaction correlates significantly with the dynamics of the electron spin, driven mainly by the ZFS Hamiltonian [23]. The autocorrelation rates of both the fluctuating HFC and nuclear Zeeman (shielding) interaction Hamiltonians are small as compared to their magnitudes (in $s^{-1}$ ). Consequently, nuclear spin relaxation would be in the Redfield regime [see Eq. (4.24) of Ref. [8]] if electron spin dynamics could be omitted (as in diamagnetic relaxation). This holds also in case of the oxygen spin relaxation, discussed below.

\section{B. Electron spin relaxation in the presence of hyperfine coupling}

We extracted the relaxation data of the effective $S=1$ spin of the unpaired electrons of the $\mathrm{Ni}^{2+}$ ion. We already reported related results for this system in our earlier paper [23] using the full theory without, however, considering the effect of the hyperfine coupling, which is done presently. Figure 4 shows the simulated electron magnetization decay at 0,3 , and $10 \mathrm{~T}$ values of the external magnetic field. Unlike in the case of ${ }^{1} \mathrm{H}$ relaxation, the time scale of the electronic relaxation is sufficiently fast to allow a direct, brute-force simulation of the entire decay curve of the magnetization. The electron relaxation process does not necessarily reach the asymptotic limit before the magnetization has practically vanished. However, the single-exponential decay fits quite well to the simulation results at $B>3 \mathrm{~T}$.

We fit the single-exponential decay of the form $e^{-R_{1} t}$ and $e^{-R_{2} t}$, respectively, to the simulated spin-lattice and spin-spin magnetization curves and extract the relaxation rates $R_{1}$ and $R_{2}$ for the electron spin. The results are shown in Fig. 5 as a function of the external magnetic-field strength. The figure also shows the simulated second-order relaxation rates $a_{1}^{(2)}$ and $a_{2}^{(2)}$.

According to our results (Fig. 5), the second-order relaxation rates are in excellent agreement with the full electron spin relaxation simulation. The rates are contributed to only 


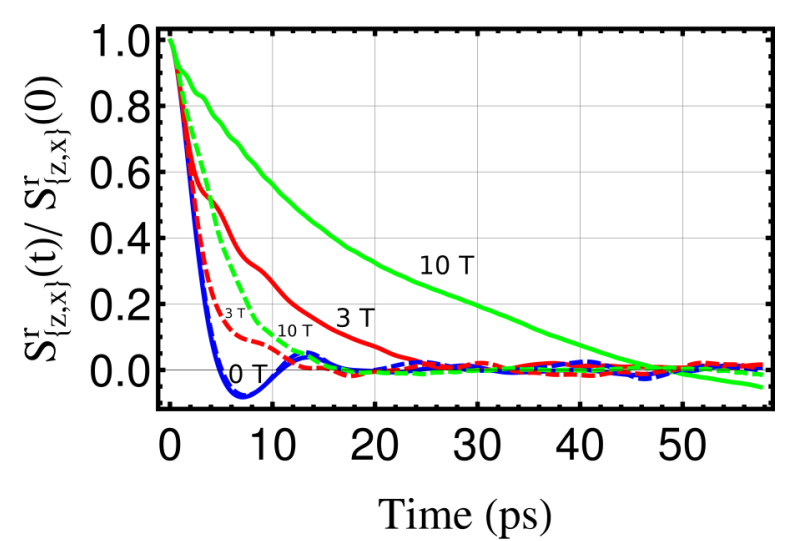

FIG. 4. Simulated electron magnetization decay $S_{z}(t) / S_{z}(0)$ (solid lines) and $S_{x}(t) / S_{x}(0)$ (dashed lines) defined by Eq. (24) at $0 \mathrm{~T}$ (blue), $3 \mathrm{~T}$ (red), and $10 \mathrm{~T}$ (green) external magnetic-field strengths, in $\mathrm{Ni}^{+2}(a q)$ at $300 \mathrm{~K}$.

by ZFS interaction, due to the diagonal assumption. The correction term [latter term in Eq. (25)] has the magnitude of circa $0.05 \times 10^{-12} \mathrm{~s}^{-1}$ for both $a_{1}^{(2)}$ and $a_{2}^{(2)}$ and, thus, turns out to be significant in the case of electron spin relaxation. As compared to the second-order rates and our earlier results [23], where only the ZFS and electronic Zeeman interaction were included, electron relaxation is to a good approximation unaffected by the HFC interaction with the proton. Reflecting the discussion of the inapplicability of the second-order approximation to ${ }^{1} \mathrm{H}$ relaxation (vide supra), the HFC interaction only has an off-diagonal contribution to the electron spin relaxation through the second-order term of the GCE. Hence, the excellent agreement that we find with the diagonal assumption suggests that such second-order HFC contributions are not significant. Furthermore, the higherorder terms contributed to by HFC interaction are always weaker as compared to the higher-order terms contributed

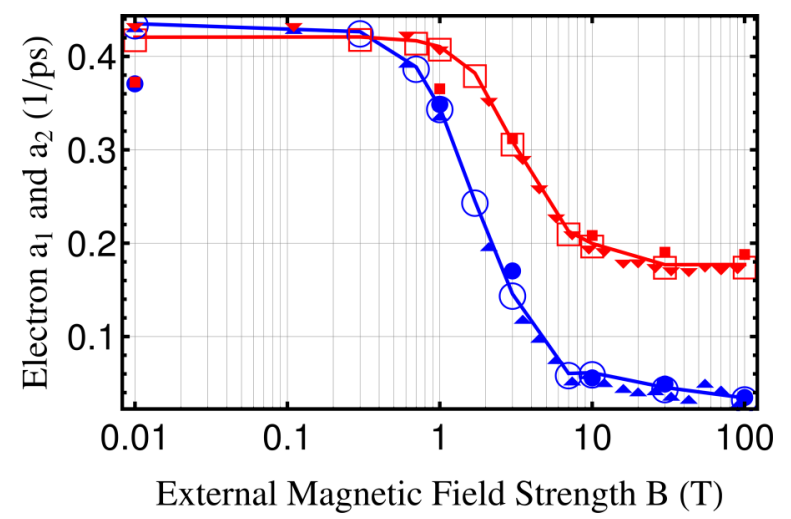

FIG. 5. Simulated electron spin-lattice ( $R_{1}$, blue open circles) and spin-spin $\left(R_{2}\right.$, red open squares) relaxation rates in $\mathrm{Ni}^{2+}(a q)$ based on the full theory as compared to the second-order rates $a_{1}^{(2)}$ (blue closed circles) and $a_{2}^{(2)}$ (red closed squares). Results as functions of the external magnetic-field strength, at $300 \mathrm{~K}$, including hyperfine coupling to proton. Proton-uncoupled $R_{1}$ and $R_{2}$ results from our earlier publication [23] are included as blue triangles pointing up $\left(R_{1}\right)$ and red triangles pointing down $\left(R_{2}\right)$.

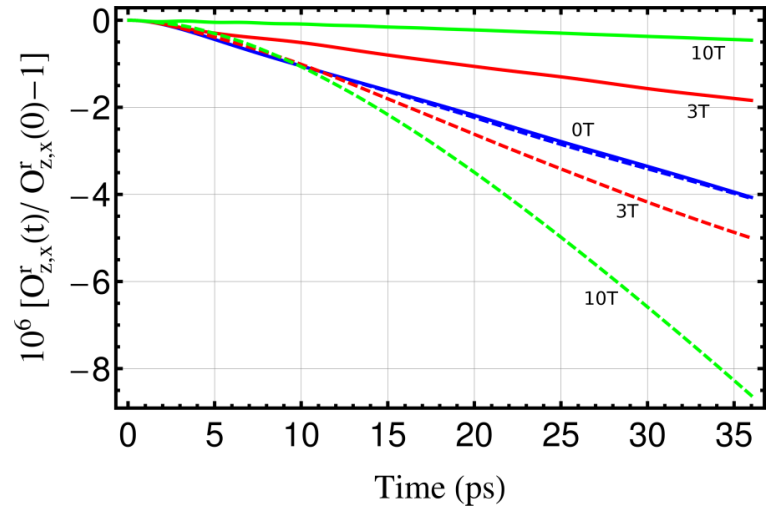

FIG. 6. Simulated oxygen magnetization decays $O_{z}^{r}(t) / O_{z}^{r}(0)$ (spin-lattice, solid lines) and $O_{x}^{r}(t) / O_{x}^{r}(0)$ (spin-spin, dashed lines) defined by Eq. (24) at $0 \mathrm{~T}$ (blue), $3 \mathrm{~T}$ (red), and $10 \mathrm{~T}$ (green) external magnetic-field strengths, at $300 \mathrm{~K}$.

solely to by the ZFS interaction. One may further surmise that the excellent agreement found with the second-order rates as compared to the full results [23] suggests that also the higher-order terms containing contributions only from ZFS are insignificant. As explained in the next section, simulations with hyperfine coupling to ${ }^{17} \mathrm{O}$ were done, due to reasons of computational cost, with lower statistical quality, which has a slight effect on the extracted electron spin relaxation rates in this case (see Supplemental Material [35]).

The autocorrelation function of the ZFS Hamiltonian [defined as $\hat{\boldsymbol{S}} \cdot \boldsymbol{D}(t) \cdot \hat{\boldsymbol{S}}$, with the magnitude of $\omega=1.4 \times 10^{12}$ $\mathrm{rad} / \mathrm{s}$ ] decays biexponentially with integrated correlation time $\tau_{c}^{\mathrm{ZFS}}=0.5 \mathrm{ps}$. Thus, $\omega \tau_{c}^{\mathrm{ZFS}}=0.74$ and the process does not occur in the Redfield regime, which is characterized by $\omega \tau_{c}^{Z F S} \ll 1$ [see Eq. (4.24) of Ref. [8]]. Nevertheless, the (Redfield-type) second-order theory describes the electron spin relaxation sufficiently. As discussed in Ref. [11], the magnitude of each successive term in the GCE can be anticipated to decrease, roughly, at least by the factor of $\omega \tau_{c}^{\text {ZFS }}$.

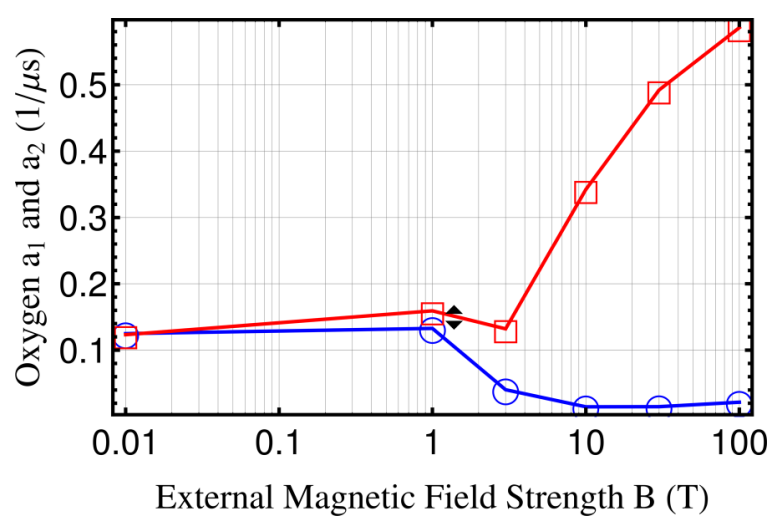

FIG. 7. Simulated $a_{1}$ (spin-lattice, blue open circles) and $a_{2}$ (spinspin, red open squares) relaxation rates of ${ }^{17} \mathrm{O}$ nuclear spin of the first solvation-shell water molecules in $\mathrm{Ni}^{2+}(a q)$. Results as functions of the external magnetic-field strength, at $300 \mathrm{~K}$. The black triangles represent experimental $a_{2}$ results interpreted from Fig. 1 of Ref. [36] [approximately $0.143(\mu \mathrm{s})^{-1}$ at $270 \mathrm{~K}$ and $0.167(\mu \mathrm{s})^{-1}$ at $300 \mathrm{~K}$ ]. 
TABLE I. Simulated full and second-order relaxation rates of the first-shell nuclear spins in $\mathrm{Ni}^{2+}(a q)$, at $300 \mathrm{~K}$.

\begin{tabular}{|c|c|c|c|c|c|c|c|c|c|c|}
\hline$B(\mathrm{~T})$ & 0 & 0.3 & 0.7 & 1.0 & 1.7 & 3 & 7 & 10 & 30 & 100 \\
\hline Proton $a_{1} \mathrm{~ms}^{-1}$ & 3.95 & 3.95 & 4.66 & 4.68 & 4.59 & 5.03 & 6.22 & 6.44 & 8.29 & 9.54 \\
\hline Proton $a_{2} \mathrm{~ms}^{-1}$ & 3.96 & 3.70 & 4.78 & 4.85 & 4.52 & 5.29 & 6.82 & 7.03 & 8.19 & 11.3 \\
\hline Proton $a_{1}^{(2)} \mathrm{ms}^{-1}$ & 46.7 & & & 18.8 & & 17.44 & & 16.0 & 13.0 & 8.01 \\
\hline Proton $a_{2}^{(2)} \mathrm{ms}^{-1}$ & 46.7 & & & 15.7 & & 14.6 & & 13.7 & 12.3 & 10.6 \\
\hline Oxygen $a_{1} \mu \mathrm{s}^{-1}$ & 0.13 & & & 0.13 & & 0.04 & & 0.02 & 0.02 & 0.02 \\
\hline Oxygen $a_{2} \mu \mathrm{s}^{-1}$ & 0.12 & & & 0.16 & & 0.13 & & 0.34 & 0.49 & 0.59 \\
\hline Oxygen $a_{1}^{(2)} \mu \mathrm{s}^{-1}$ & 0.13 & & & 0.02 & & 0.02 & & 0.02 & & 0.02 \\
\hline Oxygen $a_{2}^{(2)} \mu \mathrm{s}^{-1}$ & 0.09 & & & 0.02 & & 0.02 & & 0.02 & & 0.02 \\
\hline
\end{tabular}

Our results indicate that the magnitudes can decrease by a significantly greater factor.

\section{Relaxation of the water oxygens}

In the case of the ${ }^{17} \mathrm{O}$ nuclei of the first-shell water molecules, we only used one quarter of the length of the full series of Hamiltonians $\left\{\hat{H}_{i}\right\}$ for a more manageable computational expense. According to our tests [35], the behavior of the magnetization decay is stable against varying the length of the used part of the series of Hamiltonians: similar results are obtained both with the full series and one quarter of it (see Supplemental Material [35]). In contrast, the relaxation rates show greater dependence on the used sampling step.

Figure 6 shows the ${ }^{17} \mathrm{O}$ magnetization decay curves $O_{z}^{r}(t) / O_{z}^{r}(0)$ and $O_{x}^{r}(t) / O_{x}^{r}(0)$, at 0,3 , and $10 \mathrm{~T}$ external magnetic-field strengths, using the chosen sampling interval and length of the series of Hamiltonians. Similarly to the case of proton, the ${ }^{17} \mathrm{O}$ magnetization assumes the linear form $1+b_{1,2}-a_{1,2} t$ well within the time scale of the simulation. Figure 7 shows the extracted ${ }^{17} \mathrm{O}$ spin-lattice $\left(a_{1}\right)$ and spin-spin $\left(a_{2}\right)$ relaxation rates, as functions of $B$. Figure 7 also includes a comparison with the experimental first-shell spin-spin relaxation rate, interpreted from Fig. 1 of Ref. [36]. Our first-principles results are in excellent agreement with these data. The second-order approximations $a_{1,2}^{(2)}$ calculated with the entire series of Hamiltonians $\left\{\hat{H}_{i}\right\}$ are included in the Supplemental Material [35].

\section{TABULATED RELAXATION RATES}

Table I contains tabulated versions of the simulated nuclear spin relaxation rates as functions of the external magnetic-field strength. The simulated electron spin relaxation rates (without HFC) are tabulated in Ref. [23].

\section{CONCLUSIONS}

In this paper, we presented a method to extract the propagator for the time dependence of the combined electronic and nuclear spin density matrix. We used a time series of Hamiltonians spanning a time scale relevant for the molecular fluctuations contributing to paramagnetic relaxation. The Hamiltonians are obtained from a molecular dynamics simulation trajectory by quantum-chemical sampling.
We applied the method to the aqueous solution of the nickel(II) ion (spin-1 system) and obtained from first principles the spin-lattice $\left(1 / T_{1}\right)$ and spin-spin $\left(1 / T_{2}\right)$ relaxation rates for the nuclear and effective electron spin of the system. Whereas it is possible to simulate the entire electron magnetization decay curve using an adequate Hamiltonian sampling rate, the same is not feasible for the nuclear spins due to the much longer time scale. For the nuclei, the asymptotic limit proposed by Kubo was employed, rendering feasible the predictions of nuclear spin relaxation rates. Despite the limited statistics, due to which we had to assume that the nuclear relaxation is single exponential, both the electronic and nuclear relaxation rates are in good agreement with the available experimental data. Comparison of the simulated relaxation rates with the second-order (Redfield-type) approximation shows that the latter is insufficient for describing nuclear spin relaxation in the paramagnetic example system. However, in the case of electron spin relaxation, the second-order rates are in an excellent agreement with the results of the full simulation.

The simulated electron spin relaxation rates are dominated by the ZFS interaction. The ${ }^{1} \mathrm{H}$ spin-lattice relaxation rates possess the same order of magnitude as the corresponding experimental results. Furthermore, the results feature the experimentally observed qualitative features of the low-field plateau in the proton relaxation rates, as well as a rapid increase when approaching $10 \mathrm{~T}$. A less-pronounced minimum at the field of a few Tesla is obtained in the simulation than what is observed experimentally, however. It is thinkable that this deficiency is due to limited statistical sampling. The second-order relaxation rates are in this case of the order of 10 times higher than the rates extracted from the full simulation, at the experimentally relevant external magnetic field strengths. The higher the external magnetic field is, the better the second-order approximation becomes, and at 100-T field the results coalesce, meaning that the second-order term in the underlying generalized cumulant expansion is the highest and only significant term at this field strength. A very good agreement is found for the relaxation rates of the water oxygen with the limited amount of available data.

Quantitative, first-principles understanding of macroscopic properties is one of the aims of the molecular theory and modeling. In this paper, the PRE phenomenon was simulated from first principles, by combining spin dynamics and molecular dynamics simulations sampled through quantum-chemical techniques. More efficient QC sampling techniques, such as 
the preparametrization of the magnetic property hypersurfaces, are required to push forward the development of the present type of first-principles simulation techniques, including the understanding of the behavior of the terms in the generalized cumulant expansion.

\section{ACKNOWLEDGMENTS}

The authors are grateful to Dr. Pär Håkansson for useful discussions and Dr. Jiří Mareš for providing the magnetic property tensors. J.R. is grateful to Dr. Ilya Kuprov for a most informative spin dynamics lecture course. The research leading to these results has received funding from the People Programme (Marie Curie Actions) of the European Union's Seventh Framework Programme (No. FP7/2007-2013) under REA Grant Agreement No. 317127. Further financial support has been obtained from the Exactus doctoral program of the University of Oulu Graduate School (J.R.), and the Academy of Finland. Computational resources due to CSC-IT Center for Science (Espoo, Finland) and the Finnish Grid Initiative project, were used.

\section{APPENDIX A: ASYMPTOTIC LIMIT}

We start from Eq. (14) and note that, at $t>\tau_{i}=(i-1) \tau$, $\hat{\hat{K}}_{i}^{r}(t)$ can be split in two parts:

$$
\begin{aligned}
\left.\hat{\hat{K}}_{i}^{r}(t)\right|_{t>\tau_{i}}= & \int_{0}^{\tau_{i}} d t_{i} \int_{0}^{t_{i}} d t_{i-1} \ldots \int_{0}^{t_{2}} d t_{1}\left\langle\hat{\hat{L}}_{I}^{r}\left(t_{i}\right) \ldots \hat{\hat{L}}_{I}^{r}\left(t_{1}\right)\right\rangle_{c} \\
& +\int_{\tau_{i}}^{t} d t_{i} \int_{0}^{t_{i}} d t_{i-1} \ldots \int_{0}^{t_{2}} d t_{1}\left\langle\hat{\hat{L}}_{I}^{r}\left(t_{i}\right) \ldots \hat{\hat{L}}_{I}^{r}\left(t_{1}\right)\right\rangle_{c},
\end{aligned}
$$

where the latter part can be expressed as $\left(t-\tau_{i}\right) \frac{\partial \hat{K}_{i}^{r}(t)}{\partial t}$. In the following, we express the explicit time derivative $\frac{\partial}{\partial t}$, with a dot, i.e., $\dot{\hat{\hat{K}}}_{i}^{r}(t) \equiv \frac{\partial \hat{\hat{K}}_{i}^{r}(t)}{\partial t}$. Now, at $t>\tau_{i}$, the derivative can be expressed as

$$
\begin{aligned}
\left.\dot{\hat{\hat{K}}}_{i}^{r}(t)\right|_{t>\tau_{i}}= & \int_{t-\tau}^{t} d t_{i-1} \int_{t_{i-1}-\tau}^{t_{i-1}} d t_{i-2} \int_{t_{i-2}-\tau}^{t_{i-2}} d t_{i-3} \ldots \\
& \times \int_{t_{2}-\tau}^{t_{2}} d t_{1}\left\langle\hat{\hat{L}}_{I}^{r}(t) \hat{\hat{L}}_{I}^{r}\left(t_{i-1}\right) \ldots \hat{\hat{L}}_{I}^{r}\left(t_{1}\right)\right\rangle_{c} \\
= & \int_{t-\tau}^{t} d t_{i-1} \ldots \int_{t_{2}-\tau}^{t_{2}} d t_{1}\left\langle e^{-\hat{\hat{L}}_{z} t} \hat{\hat{L}}_{I}(t) e^{\hat{\hat{L}}_{z} t} e^{-\hat{\hat{L}}_{z} t_{i-1}}\right. \\
& \left.\times \hat{\hat{L}}_{I}\left(t_{i-1}\right) e^{\hat{\hat{L}}_{z} t_{i-1}} \ldots e^{-\hat{\hat{L}}_{z} t_{i}} \hat{\hat{L}}_{I}\left(t_{1}\right) e^{\hat{\hat{L}}_{z} t_{1}}\right\rangle_{c} .
\end{aligned}
$$

Due to the nature of the average, taken over the stationary ensemble, $\dot{\hat{\hat{K}}}_{i}^{r}(t)$ has no explicit time dependence at $t>\tau_{i}$. However, there is implicit time dependence due to the rotating frame. In the second row of Eq. (A2), this has been emphasized by writing the rotation superoperators explicitly. The rotation phase difference between the adjacent Hamiltonians, such as $e^{\hat{\hat{L}}_{z} t_{j}} e^{-\hat{\hat{L}}_{z} t_{j-1}}$ (including the first one from the left: $e^{\hat{\hat{L}}_{z} t} e^{-\hat{\hat{L}}_{z} t_{i-1}}$ ), are independent under the time translation $t \rightarrow \tau_{i}$. The first and last rotation operators $e^{-\hat{L}_{z} t}$ and $e^{\hat{\hat{L}}_{z} t_{1}}$, respectively, change under the time translation and, thus, are the only time dependence that can not be translated from $t$ to $\tau_{i}$ without influence. Thus, we see that

$$
\begin{aligned}
\left.\dot{\hat{K}}_{i}^{r}(t)\right|_{t>\tau_{i}}= & e^{-\hat{\hat{L}}_{z}\left(t-\tau_{i}\right)}\left[\int_{\tau_{i}-\tau}^{\tau_{i}} d t_{i-1} \int_{t_{i-1}-\tau}^{t_{i-1}} d t_{i-2} \ldots\right. \\
& \left.\times \int_{t_{2}-\tau}^{t_{2}} d t_{1}\left\langle\hat{\hat{L}}_{I}^{r}\left(\tau_{i}\right) \ldots \hat{\hat{L}}_{I}^{r}\left(t_{1}\right)\right\rangle_{c}\right] e^{\hat{\hat{L}}_{z}\left(t-\tau_{i}\right)} \\
= & e^{-\hat{\hat{L}}_{z}\left(t-\tau_{i}\right)} \dot{\hat{\hat{K}}}_{i}^{r}\left(\tau_{i}\right) e^{\hat{\hat{L}}_{z}\left(t-\tau_{i}\right)}
\end{aligned}
$$

where $e^{-\hat{\hat{L}}_{z}\left(t-\tau_{i}\right)}$ and $e^{\hat{\hat{L}}_{z}\left(t-\tau_{i}\right)}$ are explicitly written timedependent factors, the only time dependence remaining in $\dot{\hat{\hat{K}}}_{i}^{r}(t)$ when $t>\tau_{i}$. If $\hat{\hat{K}}_{j}^{r}(t)$ is the highest significant term in the GCE, we see that

$$
\begin{aligned}
\left.\hat{\mathscr{L}}_{I}^{r}(t)\right|_{t>\tau_{j}}= & e^{-\hat{\hat{L}}_{z}\left(t-\tau_{j}\right)} \dot{\hat{\mathscr{L}}}_{I}^{r}\left(\tau_{j}\right) e^{\hat{\hat{L}}_{z}\left(t-\tau_{j}\right)} t \\
& +\left[\hat{\hat{\mathscr{L}}}_{I}^{r}\left(\tau_{j}\right)-e^{-\hat{\hat{L}}_{z}\left(t-\tau_{j}\right)} \dot{\hat{\mathscr{L}}}_{I}^{r}\left(\tau_{j}\right) e^{\hat{\hat{L}}_{z}\left(t-\tau_{j}\right)} \tau_{j}\right] \\
\equiv & \hat{\mathscr{A}} t+\hat{\hat{\mathscr{B}}} .
\end{aligned}
$$

Both $\hat{\mathscr{A}}$ and $\hat{\hat{\mathscr{B}}}$ are explicitly time independent.

\section{APPENDIX B: LENGTH OF THE TIME STEP}

The GCE of $\hat{\mathscr{L}}_{I}^{r}(t)$ can, alternatively to the Eq. (9), be written as

$$
\begin{aligned}
\hat{\mathscr{L}}_{I}^{r}(t)= & \lim _{\max \left(\delta_{i}\right) \rightarrow 0}\left\langle e^{\hat{\hat{L}}_{I}^{r}\left(t_{n}\right) \delta_{n}} \ldots\right. \\
& \left.\times e^{\hat{\hat{L}}_{I}^{r}\left(t_{2}\right) \delta_{2}} e^{\hat{\hat{L}}_{I}^{r}\left(t_{1}\right) \delta_{1}}-\hat{\hat{1}}\right\rangle_{c} .
\end{aligned}
$$

When $\max \left(\delta_{i}\right)$ is close enough to zero, the contribution from the second and higher orders is not significant and, thus,

$$
\begin{aligned}
\hat{\hat{L}}_{I}^{r}(t)= & \lim _{\max \left(\delta_{i}\right) \rightarrow 0}\left\langle\left[\hat{\hat{1}}+\hat{\hat{L}}_{I}^{r}\left(t_{n}\right) \delta_{n}\right] \ldots\right. \\
& \left.\times\left[\hat{\hat{1}}+\hat{\hat{L}}_{I}^{r}\left(t_{2}\right) \delta_{2}\right]\left[\hat{\hat{1}}+\hat{\hat{L}}_{I}^{r}\left(t_{1}\right) \delta_{1}\right]-\hat{\hat{1}}\right\rangle_{c} .
\end{aligned}
$$

In the practical simulations we choose an equal length $\delta$ for the time steps $\delta_{i}$, small enough to catch all the significant fluctuations contributing to relaxation. With such a choice of $\delta$, the statistical quality of the simulation would not significantly further improve by choosing an even smaller time step. Now, the calculated $\hat{\mathscr{L}}_{I}^{r}$ has the form

$$
\begin{aligned}
\hat{\mathscr{L}}_{I}^{r}(n \delta)= & \left\langle\left[\hat{\hat{1}}+\hat{\hat{L}}_{I}^{r}(n \delta) \delta\right] \ldots\right. \\
& \left.\times\left[\hat{\hat{1}}+\hat{\hat{L}}_{I}^{r}(2 \delta) \delta\right]\left[\hat{\hat{1}}+\hat{\hat{L}}_{I}^{r}(\delta) \delta\right]-\hat{\hat{1}}\right\rangle_{c},
\end{aligned}
$$

and the terms in the GCE, $\hat{\mathscr{L}}_{I}^{r}(t)=\sum_{i=1}^{\infty} \hat{\hat{K}}_{i}^{r}(t)$, can be expressed as

$$
\begin{aligned}
\hat{\hat{K}}_{i}^{r}(n \delta)= & \delta^{i} \sum_{m_{i}=i}^{n} \sum_{m_{i-1}=i-1}^{m_{i}-1} \ldots \\
& \times \sum_{m_{1}=1}^{m_{2}-1}\left\langle\hat{\hat{L}}_{I}^{r}\left(m_{i} \delta\right) \hat{\hat{L}}_{I}^{r}\left(m_{i-1} \delta\right) \ldots \hat{\hat{L}}_{I}^{r}\left(m_{1} \delta\right)\right\rangle_{c} .
\end{aligned}
$$

In paramagnetic systems, the magnitude of the interaction can, however, be so large that the Taylor series expansion of $e^{\hat{\hat{L}}_{I}(t) t}$ 
cannot be truncated after the first-order term. For example, the inclusion of the second-order terms, as in

$$
\hat{\mathscr{L}}_{I}^{r}(n \delta)=\left\langle\left[\hat{\hat{1}}+\hat{\hat{L}}_{I}^{r}(n \delta) \delta+\frac{1}{2 !} \hat{\hat{L}}_{I}^{r}(n \delta)^{2} \delta^{2}\right] \ldots\left[\hat{\hat{1}}+\hat{\hat{L}}_{I}^{r}(\delta) \delta+\frac{1}{2 !} \hat{\hat{L}}_{I}^{r}(\delta)^{2} \delta^{2}\right]-\hat{\hat{1}}\right\rangle_{c},
$$

would also introduce second-order terms (with respect to the same time step) to GCE:

$$
\frac{\delta}{2 !} \sum_{l=1}^{n}\left[\delta^{i} \sum_{m_{i}=i}^{n} \ldots \sum_{m_{l}=l}^{m_{l+1}-1} \ldots \sum_{m_{1}=1}^{m_{2}-1}\left\langle\hat{\hat{L}}_{I}^{r}\left(m_{i} \delta\right) \ldots \hat{\hat{L}}_{I}^{r}\left(m_{l} \delta\right)^{2} \ldots \hat{\hat{L}}_{I}^{r}\left(m_{1} \delta\right)\right\rangle_{c}\right] \equiv{ }^{(2)} \hat{\hat{K}}_{i}^{r}(t) .
$$

The cumulant average in this case can be expressed as

$$
\left\langle\hat{\hat{L}}_{I}^{r}\left(t_{n}\right) \ldots \hat{\hat{L}}_{I}^{r}\left(t_{l}\right)^{2} \ldots \hat{\hat{L}}_{I}^{r}\left(t_{1}\right)\right\rangle_{c}=\left.\left[\frac{\partial}{\partial \delta_{l}} \frac{\partial^{n}}{\partial \delta_{1} \ldots \partial \delta_{n}} \ln \left\langle e^{\hat{\hat{L}}_{I}^{r}\left(t_{n}\right) \delta_{n}} \ldots e^{\hat{\hat{L}}_{I}^{r}\left(t_{1}\right) \delta_{1}}\right\rangle\right]\right|_{\delta_{i}=0 \quad \forall i} .
$$

${ }^{(2)} \hat{\hat{K}}_{i}^{r}(t)$ turns asymptotic at $\tau_{i}$ and may be identified as a correction term to $\hat{\hat{K}}_{i+1}^{r}(t)$ due to the finite time step. For example, the correction to the second term $\hat{\hat{K}}_{2}^{r}(t)$ of the GCE is

$$
{ }^{(2)} \hat{\hat{K}}_{1}^{r}(n \delta)=\frac{\delta^{2}}{2 !} \sum_{m_{1}=1}^{n}\left\langle\hat{\hat{L}}_{I}^{r}\left(m_{1} \delta\right)^{2}\right\rangle_{c}=\frac{\delta^{2}}{2 !} \sum_{m_{1}=1}^{n}\left\langle\hat{\hat{L}}_{I}^{r}\left(m_{1} \delta\right)^{2}\right\rangle=\frac{\delta}{2 !}\left\langle\hat{\hat{L}}_{I}^{r}(n \delta)^{2}\right\rangle n \delta .
$$

Thus, the complete second term of the GCE reads as

$$
\begin{aligned}
\hat{\hat{K}}_{2}^{r}(n \delta) & =\delta^{2} \sum_{m_{2}=2}^{n} \sum_{m_{1}=1}^{m_{2}-1}\left\langle\hat{\hat{L}}_{I}^{r}\left(m_{2} \delta\right) \hat{\hat{L}}_{I}^{r}\left(m_{1} \delta\right)\right\rangle_{c}+\frac{\delta^{2}}{2 !} \sum_{m_{1}=1}^{n}\left\langle\hat{\hat{L}}_{I}^{r}\left(m_{1} \delta\right)^{2}\right\rangle_{c}=\delta^{2} \sum_{m_{2}=1}^{n} \sum_{m_{1}=1}^{m_{2}}\left\langle\hat{\hat{L}}_{I}^{r}\left(m_{2} \delta\right) \hat{\hat{L}}_{I}^{r}\left(m_{1} \delta\right)\right\rangle_{c}-\frac{\delta^{2}}{2} \sum_{m_{1}=1}^{n}\left\langle\hat{\hat{L}}_{I}^{r}\left(m_{1} \delta\right)^{2}\right\rangle_{c} \\
& =\delta^{2} \sum_{m_{2}=1}^{n} \sum_{m_{1}=1}^{m_{2}}\left\langle\hat{\hat{L}}_{I}^{r}\left(m_{2} \delta\right) \hat{\hat{L}}_{I}^{r}\left(m_{1} \delta\right)\right\rangle-\frac{\delta}{2}\left\langle\hat{\hat{L}}_{I}^{r}(n \delta)^{2}\right\rangle n \delta
\end{aligned}
$$

More generally, higher terms in GCE also have higher-order corrections. The third term $\hat{\hat{K}}_{3}^{r}(t)$ has one third-order correction and two second-order corrections:

$$
\begin{aligned}
\hat{\hat{K}}_{3}^{r}(t)= & \delta^{3} \sum_{m_{3}=3}^{n} \sum_{m_{2}=2}^{m_{3}-1} \sum_{m_{1}=1}^{m_{2}-1}\left\langle\hat{\hat{L}}_{I}^{r}\left(m_{3} \delta\right) \hat{\hat{L}}_{I}^{r}\left(m_{2} \delta\right) \hat{\hat{L}}_{I}^{r}\left(m_{1} \delta\right)\right\rangle_{c}+\frac{\delta^{3}}{3 !} \sum_{m_{1}=1}^{n}\left\langle\hat{\hat{L}}_{I}^{r}\left(m_{i} \delta\right)^{3}\right\rangle_{c} \\
& +\frac{\delta^{3}}{2 !} \sum_{m_{2}=2}^{n} \sum_{m_{1}=1}^{m_{2}-1}\left[\left\langle\hat{\hat{L}}_{I}^{r}\left(m_{2} \delta\right)^{2} \hat{\hat{L}}_{I}^{r}\left(m_{1} \delta\right)\right\rangle_{c}+\left\langle\hat{\hat{L}}_{I}^{r}\left(m_{2} \delta\right) \hat{\hat{L}}_{I}^{r}\left(m_{1} \delta\right)^{2}\right\rangle_{c}\right],
\end{aligned}
$$

and the fourth term with the corrections reads as

$$
\begin{aligned}
& \hat{\hat{K}}_{4}^{r}(t) \\
& =\delta^{4} \sum_{m_{4}=4}^{n} \sum_{m_{3}=3}^{m_{4}-1} \sum_{m_{2}=2}^{m_{3}-1} \sum_{m_{1}=1}^{m_{2}-1}\left\langle\hat{\hat{L}}_{I}^{r}\left(t_{4}\right) \hat{\hat{L}}_{I}^{r}\left(t_{3}\right) \hat{\hat{L}}_{I}^{r}\left(t_{2}\right) \hat{\hat{L}}_{I}^{r}\left(t_{1}\right)\right\rangle_{c}+\frac{\delta^{4}}{4 !} \sum_{m_{1}=1}^{n}\left\langle\hat{\hat{L}}_{I}^{r}\left(m_{1} \delta\right)^{4}\right\rangle_{c}+\frac{\delta^{4}}{3 !} \sum_{m_{2}=2}^{n} \sum_{m_{1}=1}^{m_{2}-1}\left[\left\langle\hat{\hat{L}}_{I}^{r}\left(m_{2} \delta\right)^{3} \hat{\hat{L}}_{I}^{r}\left(m_{1} \delta\right)\right\rangle_{c} \quad(\mathrm{~B} 12)\right. \\
& \left.\quad+\left\langle\hat{\hat{L}}_{I}^{r}\left(m_{2} \delta\right) \hat{\hat{L}}_{I}^{r}\left(m_{1} \delta\right)^{3}\right\rangle_{c}\right]+\frac{\delta^{4}}{2 ! 2 !} \sum_{m_{2}=2}^{n} \sum_{m_{1}=1}^{m_{2}-1}\left\langle\hat{\hat{L}}_{I}^{r}\left(m_{2} \delta\right)^{2} \hat{\hat{L}}_{I}^{r}\left(m_{1} \delta\right)^{2}\right\rangle_{c}+\frac{\delta^{4}}{2 !} \sum_{m_{3}=3}^{n} \sum_{m_{2}=2}^{m_{3}-1} \sum_{m_{1}=1}^{m_{2}-1}\left[\left\langle\hat{\hat{L}}_{I}^{r}\left(m_{3} \delta\right)^{2} \hat{\hat{L}}_{I}^{r}\left(m_{2} \delta\right) \hat{\hat{L}}_{I}^{r}\left(m_{1} \delta\right)\right\rangle_{c}\right. \\
& \left.\quad+\left\langle\hat{\hat{L}}_{I}^{r}\left(m_{3} \delta\right) \hat{\hat{L}}_{I}^{r}\left(m_{2} \delta\right)^{2} \hat{\hat{L}}_{I}^{r}\left(m_{1} \delta\right)\right\rangle_{c}+\left\langle\hat{\hat{L}}_{I}^{r}\left(m_{3} \delta\right) \hat{\hat{L}}_{I}^{r}\left(m_{2} \delta\right) \hat{\hat{L}}_{I}^{r}\left(m_{1} \delta\right)^{2}\right\rangle_{c}\right] .
\end{aligned}
$$

[1] J. Kowalewski, Nucl. Magn. Reson. 40, 205 (2011).

[2] A. Schweiger and G. Jeschke, Principles of Pulse Electron Paramagnetic Resonance (Oxford University Press, Oxford, 2001).

[3] J. E. Harriman, Theoretical Foundations of Electron Spin Resonance (Academic, New York, 1978).
[4] I. Bertini, C. Luchinat, and G. Parigi, Solution NMR of Paramagnetic Molecules: Applications to Metallobiomolecules and Models (Elsevier, Amsterdam, 2001).

[5] J. Kowalewski, D. Kruk, and G. Parigi, Adv. Inorg. Chem. 57, 41 (2005). 
[6] E. A. Waters and S. A. Wickline, Basic Res. Cardiol. 103, 114 (2008).

[7] J. Iwahara and G. M. Clore, Nature (London) 440, 1227 (2006).

[8] J. Kowalewski and L. Mäler, Nuclear Spin Relaxation in Liquids: Theory, Experiments, and Applications (Taylor and Francis, London, 2006).

[9] A. G. Redfield, IBM J. Res. Dev. 1, 19 (1957).

[10] R. K. Wangsness and F. Bloch, Phys. Rev. 89, 728 (1953).

[11] R. Kubo, J. Math. Phys. 4, 174 (1963).

[12] J. H. Freed, G. Bruno, and C. F. Polnaszek, J. Chem. Phys. 75, 3385 (1971).

[13] N. Benetis, J. Kowalewski, L. Nordenskiöld, H. Wennerström, and P.-O. Westlund, Mol. Phys. 48, 329 (1983).

[14] T. Nilsson and J. Kowalewski, J. Magn. Reson. 146, 345 (2000).

[15] D. Kruk, J. Kowalewski, D. S. Tipikin, J. H. Freed, M. Mościcki, A. Mielczarek, and M. Port, J. Chem. Phys. 134, 024508 (2011).

[16] M. Odelius, C. Ribbing, and J. Kowalewski, J. Chem. Phys. 103, 1800 (1995).

[17] N. Usova, G. Bruno, P.-O. Westlund, and I. I. Fedchenia, J. Chem. Phys. 103, 96 (1995).

[18] P. Håkansson, P.-O. Westlund, E. Lindahl, and O. Edholm, Phys. Chem. Chem. Phys. 3, 5311 (2001).

[19] P. Håkansson and P. Nair, Phys. Chem. Chem. Phys. 13, 9578 (2011).

[20] H. G. Hertz and M. Holz, J. Magn. Reson. (1969) 63, 64 (1985).

[21] J. Kowalewski, T. Larsson, and P.-O. Westlund, J. Magn. Reson. (1969) 74, 56 (1987).

[22] K. Åman and P.-O. Westlund, Mol. Phys. 102, 1085 (2004).

[23] J. Rantaharju, J. Mareš, and J. Vaara, J. Chem. Phys. 141, 014109 (2014).
[24] R. Kubo, J. Phys. Soc. Jpn. 17, 1100 (1962).

[25] J. H. Freed, J. Chem. Phys. 49, 376 (1968).

[26] J. Mareš, M. Hanni, P. Lantto, J. Lounila, and J. Vaara, Phys. Chem. Chem. Phys. 16, 6916 (2014).

[27] P. Håkansson (unpublished).

[28] T. S. Pennanen, J. Vaara, P. Lantto, A. Sillanpää, K. Laasonen, and J. Jokisaari, J. Am. Chem. Soc. 126, 11093 (2004).

[29] J. Jeener, Adv. Magn. Reson. 10, 1 (1982).

[30] J. J. Sakurai, in Modern Quantum Mechanics, 2nd ed., edited by J. Napolitano (Addison-Wesley, Boston, 2010).

[31] C. W. Gardiner, Handbook of Stochastic Methods, 2nd ed. (Springer, Berlin, 1985).

[32] M. H. Levitt, SPINDYNAMICA, with contributions by J. Rantaharju, A. Brinkmann, and S. S. Roy (see www.SpinDynamica.soton.ac.uk).

[33] I. Kuprov, University of Southampton, Spin Dynamics Lecture Course, www.spindynamics.org.

[34] Wolfram Research, Inc. Champaign, IL, Mathematica version 9.0.1 (2013).

[35] See Supplemental Material at http://link.aps.org/supplemental/ 10.1103/PhysRevA.94.043413 for the calculated spin-lattice and spin-spin relaxation rates of the individual protons in the first solvation shell of the $\mathrm{Ni}^{2+}$ ion, calculated spin-lattice and spin-spin relaxation rates of the electron spin under the influence of the hyperfine coupling to the water oxygen, investigation of the effects of the Hamiltonian sampling interval and length of the used part of the series of Hamiltonians on the ${ }^{17} \mathrm{O}$ magnetization decay curves, and the extracted ${ }^{17} \mathrm{O}$ second-order relaxation rates.

[36] J. W. Neely and R. E. Connick, J. Am. Chem. Soc. 94, 3419 (1972). 